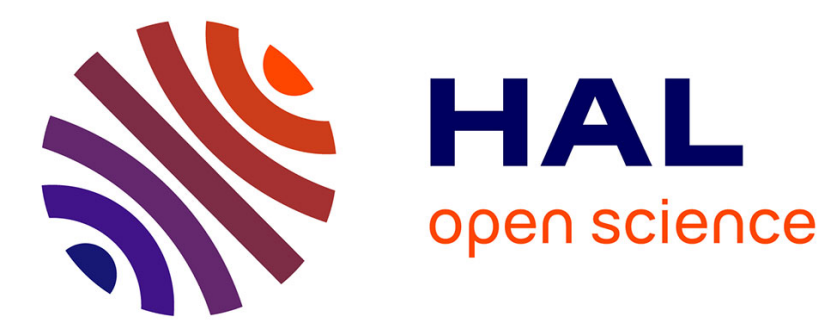

\title{
Improving the Film Cooling of a Rotor Blade Platform
}

Giovanna Barigozzi, Antonio Perdichizzi, Roberto Abram

\section{To cite this version:}

Giovanna Barigozzi, Antonio Perdichizzi, Roberto Abram. Improving the Film Cooling of a Rotor Blade Platform. 16th International Symposium on Transport Phenomena and Dynamics of Rotating Machinery (ISROMAC 2016), Apr 2016, Honolulu, United States. hal-01517887

\section{HAL Id: hal-01517887 \\ https://hal.science/hal-01517887}

Submitted on 3 May 2017

HAL is a multi-disciplinary open access archive for the deposit and dissemination of scientific research documents, whether they are published or not. The documents may come from teaching and research institutions in France or abroad, or from public or private research centers.
L'archive ouverte pluridisciplinaire HAL, est destinée au dépôt et à la diffusion de documents scientifiques de niveau recherche, publiés ou non, émanant des établissements d'enseignement et de recherche français ou étrangers, des laboratoires publics ou privés. 


\title{
Improving the Film Cooling of a Rotor Blade Platform
}

\author{
Giovanna Barigozzi ${ }^{1}$, Antonio Perdichizzi ${ }^{*}$, Roberto Abram ${ }^{2}$
}

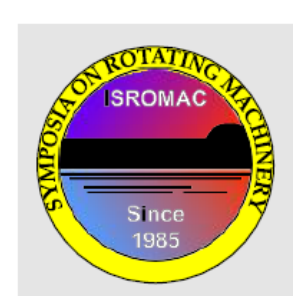

ISROMAC 2016

International

Symposium on

Transport

Phenomena and

Dynamics of

Rotating Machinery

Hawaii, Honolulu

April 10-15, 2016

\section{Abstract}

This paper shows the results of an experimental activity developed in cooperation between Ansaldo Energia and the Department of Engineering and Applied Science of Bergamo University with the aim of assessing the impact of newly designed holes on the thermal protection of a rotor blade platform. The original rotor blade platform featured 10 cylindrical holes located along the blade pressure side. Moreover, the channel front side was cooled exploiting the seal purge flow exiting the stator to rotor interface gap. The front mid channel, and particularly the region around the inter-platform gap, remained uncooled. To protect this region two sets of cylindrical holes were designed and manufactured on a 7 blade cascade model for experimental verification. Aerodynamic and thermal tests were carried out at low Mach number. To evaluate the interaction of injected flow with secondary flows a 5hole probe was traversed downstream of the trailing edge plane. The thermal behavior was analyzed by using Thermochromic Liquid Crystals technique, so to obtain film cooling effectiveness distributions. The 7-hole configuration coupled with a low blowing ratio of about 1.0 provided the best thermal protection without any impact on the aerodynamic performance.

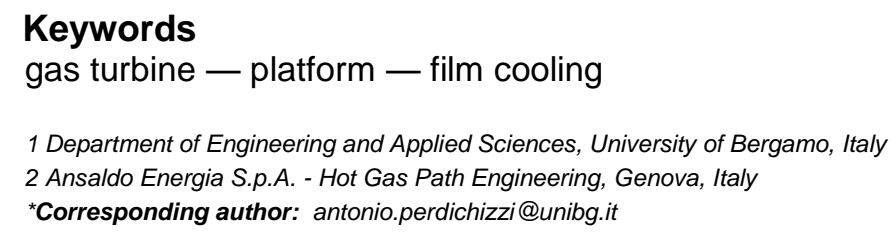

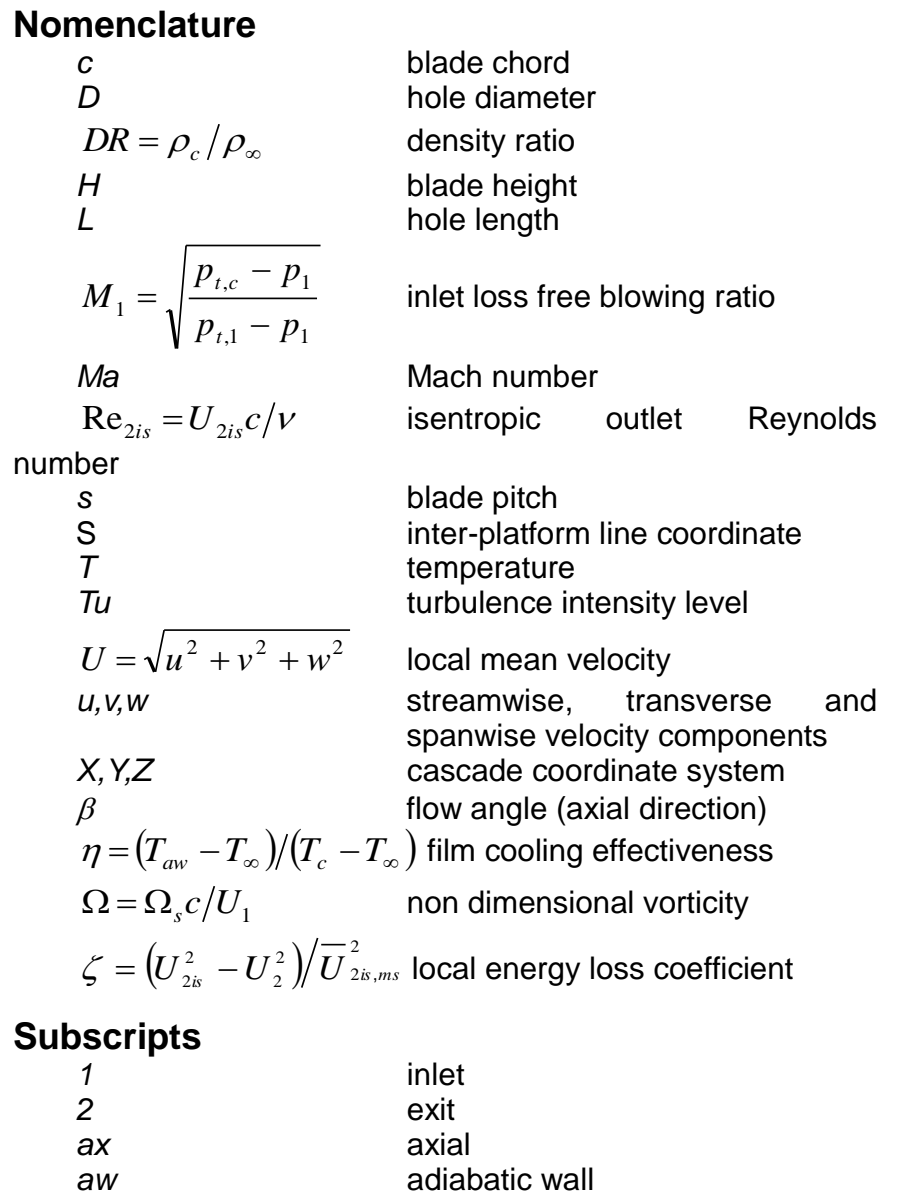

cooling flow isentropic condition streamwise free stream

\section{INTRODUCTION}

In modern gas turbines the achievement of high performance requires a continuous increase of turbine inlet temperature. Hence an enhancement of thermal protection of all surfaces directly exposed to the hot gases, including platform regions, is needed.

Many researchers have investigated film cooling on vane end walls, both through upstream slots [1-3] and discrete holes within the passage [4-10]. From the above studies, it is found that film cooling is strongly affected by end wall secondary flows. The cross flow tends to transport the injected coolant from the pressure side to the suction side of the passage. Only few works in the open literature deal with film cooling of rotor end walls. Blair [11] deeply analysed the effects of flow three dimensionality on the heat transfer in a rotating blade row. The flow structure in the rotor blade passage has been investigated by Wang et al [12]. Goldstein and Spores [13] detected high heat transfer near the bladeend wall junctions of the passage, due to an intense activity of corner vortices. Other research activities are focused on the analysis of the film cooling effectiveness due to purging of sealing flow through the stator-rotor axial gap. The areas that are typically difficult to cool, 
i.e. the region near the leading edge, can be cooled effectively with the upstream slot injection. Gao et al. [14] and Narzary [15] tested a typical labyrinth-like stator-rotor seal under different blowing conditions, looking for the thermal protection capability of the purge flow. Papa et al. [16] performed heat transfer measurements and flow visualizations for different blowing ratios in a linear rotor with an injection slot upstream of rotor blades. In all these papers, tests were performed on linear cascade wind tunnels with radial purge flow, i.e. without modeling the rotation effects. Barigozzi et al. [17] experimentally investigated the effect of rotation on slot platform cooling. Rotation effect was simulated in a linear cascade by installing fins inside of the slot to change the coolant to mainstream injection angle in the tangential direction. They showed that rotation has a significant impact on the 3D flow field and film cooling effectiveness, as it reinforces the secondary flows development and migration across the blade passage. Pau et al. [18] carried out a complete aero-thermal investigation on the purge and platform film cooling on a transonic turbine stage. Suryanarayanan et al. [19-20] investigated film cooling through the wheel space cavity in a rotating platform. All these studies showed how the interaction of the emerging purge flow structure with the upcoming main flow is complicated by the influence of rotation, resulting in a highly skewed flow emerging from the slot. The leading edge region of the platform can be effectively cooled by this purge flow. But such coolant flow only succeeds in cooling the front end wall part, before being captured by the passage vortex, leaving most of the channel practically uncooled. So discrete holes are usually added inside of the passage in some critical regions.

In some recent works, it has been shown that a complete film cooling protection on a rotating platform can be provided by combining upstream stator-rotor gap ejection and injection from discrete holes located in the rear part of the passage [14,21-22]. Discrete holes distributed along the pressure side can properly cool the rear part of the platform [23], but some more holes are still required to protect the mid channel front region, and particularly the inter-platform gap region.

The present paper deals with the aerodynamic and thermal behaviour of a rotor blade cascade with a discrete hole cooling scheme on the platform. Starting from the original coolant injection scheme with holes located in the rear part of the passage near to the filleted pressure side of the blade (Fig. 1a - [24]) and from the results coming from an upstream slot cooling configuration (Fig. 1b - [17]), a new set of holes was conceived to protect the blade to blade inter-platform front region, a region not covered by the aforementioned cooling schemes. The local effectiveness distribution is strongly dependent on the holes layout: the arrangement of film cooling holes on the platform is a key point. Two cooling schemes were thus designed, with an increasing number of holes.

This paper shows the results of different hole number and the effects of injection conditions on film cooling effectiveness of a rotor blade platform.

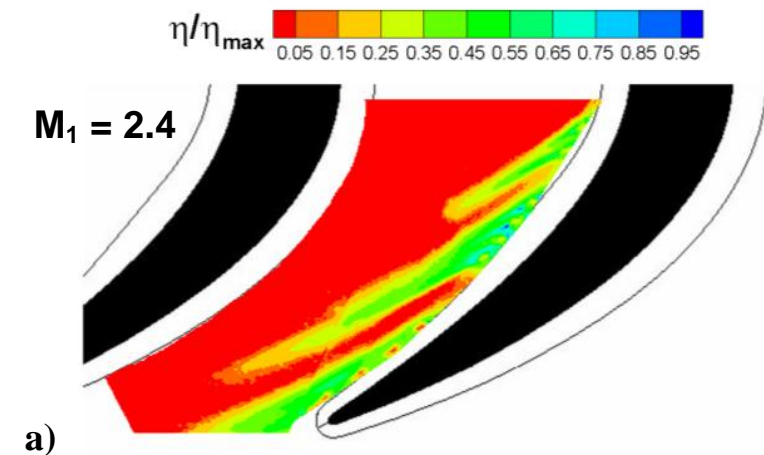

b)

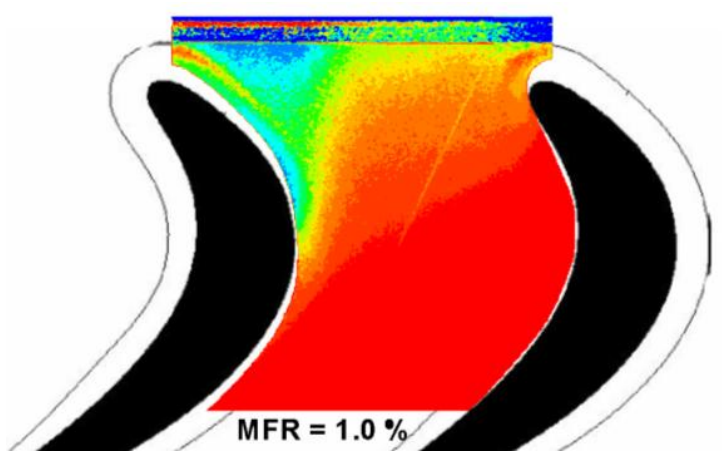

Figure. 1. Platform Film cooling effectiveness distribution: a) pressure side cooling holes [24] and b) purge gap [17].

\section{EXPERIMENTAL DETAILS}

Tests have been performed at the Turbomachinery Laboratory of the University of Bergamo in the wind tunnel for linear rotor cascades. It is a continuous running suction type wind tunnel that assures a complete optical access because entirely made up of Plexiglas (Fig. 2). The cascade model consists of a 7 blade cascade whose geometry (Fig. 2) is typical of a first high pressure turbine rotor blade. It has been tested at low Mach number $\left(M a_{2 i s}=0.3\right)$ with a low inlet turbulence intensity level $\left(T u_{1}=0.7 \%\right)$. Geometrical details of the cascade and operating conditions are all summarized in Table 1. On the hub side, the connection between the blade and the platform is realized through a 3D fillet, whose trace in the platform plane is depicted 
in Fig. 3. Only one channel is cooled. The original cooling scheme was made of ten $0.7 \mathrm{~mm}$ diameter holes located on the filleted end wall along the blade pressure side (Fig. 3). More details on this geometry are given in Barigozzi et al. [17]. On the same platform five more holes with $1.0 \mathrm{~mm}$ diameter have been initially realized. These holes are distributed along the inter-platform gap with an inclination angle of $40^{\circ}$ with respect to the end wall surface. Note that this is a quite high angle compared to those usually adopted for film cooling, but it was imposed by design constrains related to the internal gas path of the real blade. Hole length over diameter ratio $L / D$ is 11.7 . Two plenum integrated in the blade platform (Fig. 4) independently feed the two sets of holes (the 10 original holes and the new ones). Starting from the five hole configuration, a second cooling scheme was realized by adding two more holes in line with the first five (Fig. $3)$.

Injection conditions were controlled through a continuous monitoring of coolant total pressure in the two feeding chambers. The amount of the injected mass flow was in fact so small to be practically notmeasurable with a sufficiently high accuracy. The inlet loss free blowing ratio $M_{1}$ was thus used to identify each injection condition for the two sets of holes. Coolant total pressure and temperature are measured by pressure taps and T-type thermocouples located on the back side wall of each plenum. The uncertainty in the $M_{1}$ value was computed on the basis of pressure transducers $( \pm 5.5 \mathrm{~Pa})$ and Pitot probe $( \pm 10 \mathrm{~Pa})$ uncertainties. $\delta M_{1}$ resulted to be \pm 0.04 at a value of $M_{1}=1.0$ and \pm 0.08 at a value of $M_{1}=2.0$.

Aerodynamic measurements were performed $8 \%$ of the axial chord $\left(X / c_{a x}=108 \%\right)$ downstream of the trailing edge plane by using a 5-hole miniaturized aerodynamic pressure probe. The measurement plane covers two blade passages and extends over half of the blade span. The measurement grid is made of 30 points per pitch in tangential direction times 15 points along the blade height. The grid spacing was reduced approaching the end wall surface. Uncertainties in both static and total pressure have been estimated to be $\pm 0.15 \%$ of dynamic pressure.

Sprayable wide banded Thermochromic Liquid Crystals (Hallcrest BM/R25C10WC17-10) were used to get the film cooling effectiveness distributions. The surface is illuminated by means of two strips of white light LED while TLC images were acquired by using a Nikon D7100 camera. The TLC calibration was performed in situ, substituting the blade central passage with a calibration device consisting in an instrumented $\left(10 \mathrm{~T}\right.$-type thermocouples, $\left.\delta T= \pm 0.1^{\circ} \mathrm{C}\right)$ flat aluminium plate over which a temperature gradient can be generated. All calibrations and measurements were performed in the dark, in order to eliminate any influence of background illumination. Moreover, an illumination intensity as uniform as possible was provided to the model surface by properly orienting the lighting system, in the meanwhile avoiding any light reflection onto the camera.

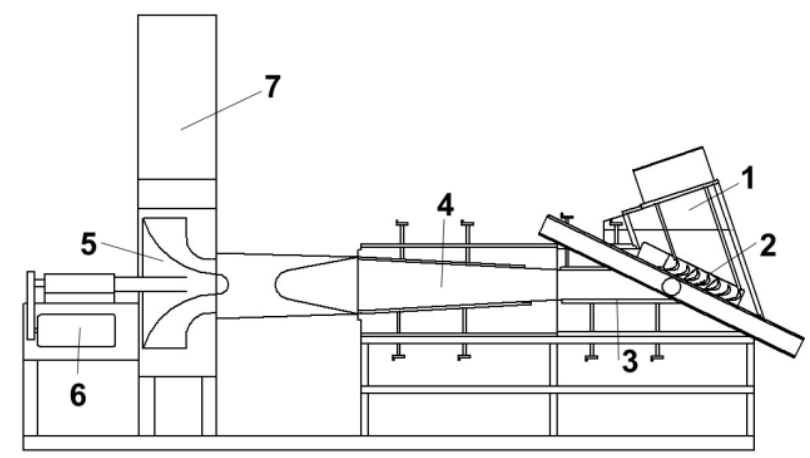

Figure 2. The wind tunnel (1: inlet duct; 2 : test section; 3 : tailboard; 4: diffuser; 5: fan; 6: AC motor; 7: discharge channel).

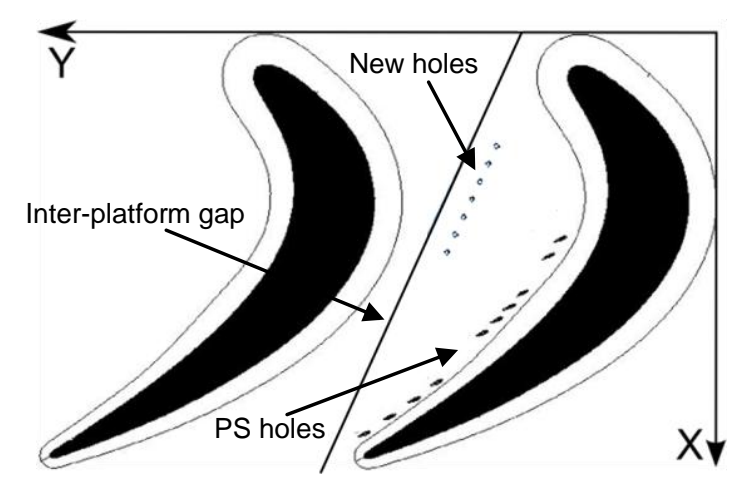

Figure 3. Cascade and endwall cooling geometry.

Table 1. Cascade geometry and operating conditions.

\begin{tabular}{|c|c|}
\hline$s / c=0.69$ & $H / c=1.26$ \\
\hline$c=116.7 \mathrm{~mm}$ & $\beta_{2}=-64.19^{\circ}$ \\
\hline$T u_{1}=0.7 \%$ & $M a_{2 i s}=0.3$ \\
\hline$M a_{1}=0.183$ & $M_{1, \text { PS }}=1-2$ \\
\hline$R e_{2 i s}=0.7410^{\circ}$ & $M_{1, \text { New }}=1-2$ \\
\hline
\end{tabular}

During tests the heated secondary flow $(D R=0.95)$ was injected into the main flow at ambient temperature. The time history of the TLC image was recorded by the camera, together with the temperature variation inside the feeding chambers $T_{c}$ and the main flow temperature $T_{\infty}$. The RGB to hue conversion [25] was applied to a single image recorded after a time period of about $60 \mathrm{~s}$, i.e. when a stable temperature level inside each plenum was reached, as well as on the end wall surface. Figure 5 is an example of an image selected. In image selection particular attention 
was paid to avoid important conduction phenomena in the most critical region, i.e. just upstream of PS hole locations. $\eta$ computation was based on the maximum temperature measured inside of each plenum, once a stable coolant temperature value was reached. The relatively large thickness of the end wall (Plexiglas made) assured to comply with wall adiabatic condition over most of the platform. Film cooling effectiveness uncertainty depends on TLC $\left(\delta T_{w}= \pm 0.3^{\circ} \mathrm{C}\right)$ and thermocouple measurements $\left(\delta T_{\infty}= \pm 0.1^{\circ} \mathrm{C}\right.$ and $\left.\delta T_{c}= \pm 0.5^{\circ} \mathrm{C}\right)$ and conduction effects. In regions where conduction phenomena do not exist, $\eta$ uncertainty will range from $\pm 5 \%$ with $\eta=$ 0.5 , up to about $\pm 15 \%$ when $\eta=0.1$. Larger uncertainty will exist if conduction phenomena become relevant. More details on thermal tests are in Barigozzi et al. [23]

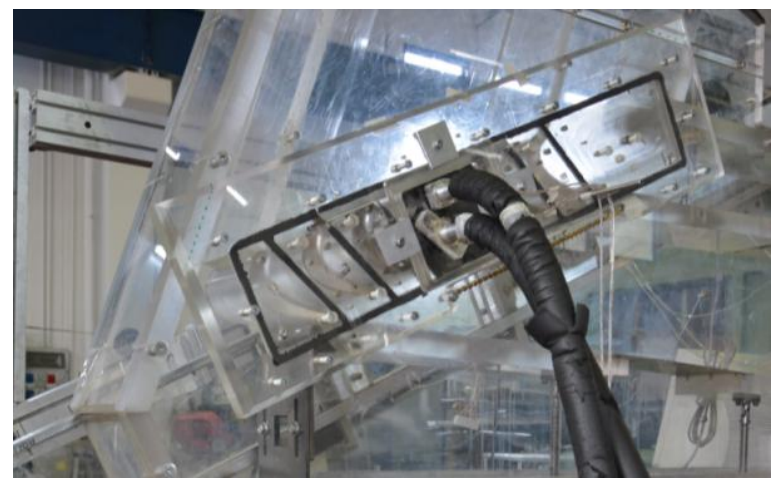

Figure 4. The cooling flow supply lines.

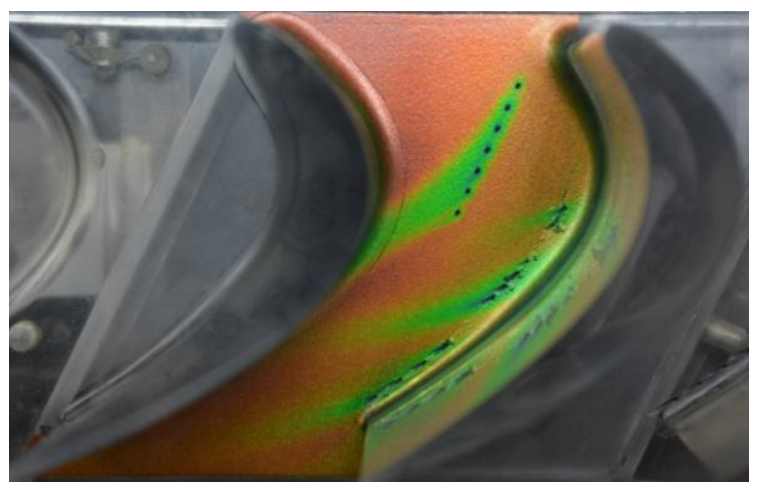

Figure 5. Example of selected image.

\section{AERODYNAMIC RESULTS}

Aerodynamic results showing the secondary flow structure have been obtained by traversing 5-hole probe $0.08 c_{a x}$ downstream of the blade trailing edge. The contour plots of energy loss coefficient $\zeta$ and stream wise vorticity $\Omega$ with secondary velocity vectors are reported in Fig. 6 , to provide the secondary flow configuration downstream of the cascade. Only the plots referred to the seven holes configuration and the minimum tested $M_{1}$ are shown, as no significant variations of secondary flow configuration were found for the other scheme or blowing conditions. It is worth noting that very similar results were also obtained testing the uncooled cascade. This essentially because the injected flow rate is always very small and insufficient to affect the secondary flows development. Moreover, coolant injection is performed downstream of the passage vortex separation line (Fig. 7); hence most of the injected flow is not captured by passage vortex and it is only subjected to the endwall cross flow from pressure to suction side.

Typical and very well defined secondary flows structures can be observed from Fig. 6 . The flow field is dominated by the presence of a well defined passage vortex, corresponding to the positive vorticity region and to the loss core on the suction side of the blade wake. The high flow turning makes the passage vortex position to be significantly shifted towards mid span. A relevant cross flow is present at the end wall, driving low energy boundary layer flow and the injected coolant from the pressure to the suction side of the vane. No trace of corner vortex loss peak can be observed, probably because it is confined in a thin layer outside of the measuring domain.
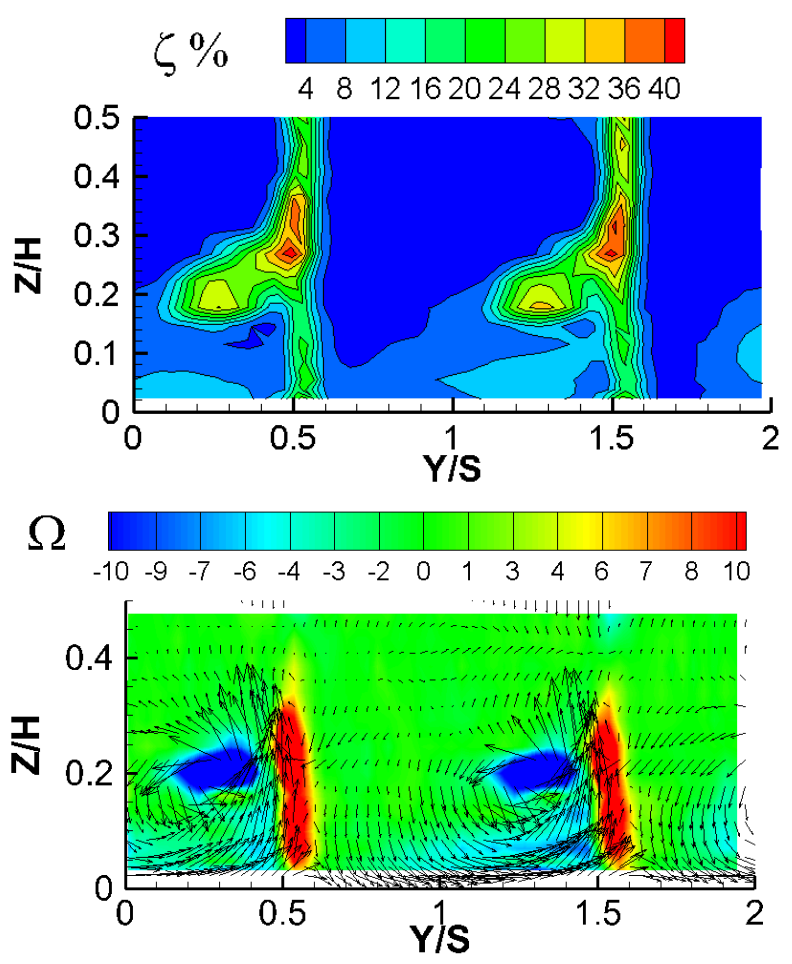

Figure 6. $\zeta$ and $\Omega$ distributions at $X / c_{a x}=108 \%$ for 7 holes $\left(M_{1, P S}=1.3\right.$ and $\left.M_{1,7 \text { holes }}=1.1\right)$. 


\section{THERMAL RESULTS}

Film cooling effectiveness distributions measured for the 5 hole cooling scheme at three different blowing conditions $\left(M_{1}\right.$ about $1.2,1.6$ and 2.0 for both PS holes and 5 new holes) are presented in Fig. 8. Data of each test have been normalized referring to the maximum measured value $\eta_{\max }$ over all tests.

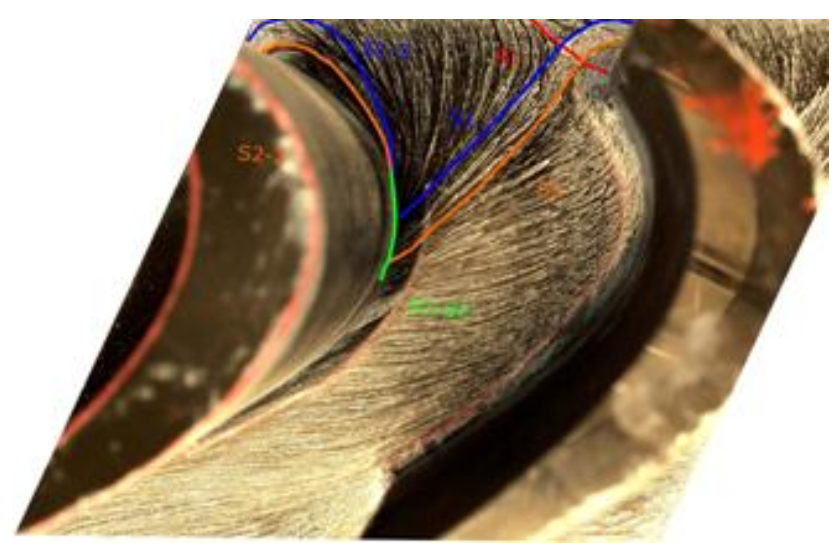

Figure 7. Oil and dye flow visualization (solid end wall).

First focusing on the performance of the new holes, an increase of inlet loss free blowing ratio results in a decrease of thermal protection. In fact, at the lowest blowing ratio (i.e. $M_{1}=1.2$ ) coolant is discharged through all the holes, especially the last one. Coolant remains attached to the wall, but its trajectory follows the three dimensional separation lines and never crosses it (see traces of these lines $S_{1}$ and $S_{2}$ on the effectiveness contour plot of Fig. 8a). Anyways, coolant persistency is good enough to allow it to almost reach the suction side of the adjacent blade. Unfortunately, the area interested by film cooling is quite limited as coolant quickly mixes with the passage vortex, hence a poor thermal coverage is attained. This is surely due to the fact that new holes are located close to the separation lines, resulting in a strong interaction between jets and passage vortex. Moreover, their injection angle is quite high $\left(40^{\circ}\right)$, resulting in a strong mixing.

Increasing the injection rate up to $M_{1}=1.6$ jet persistency downstream of the holes strongly reduces. This is due to the relatively high injection angle of $40^{\circ}$, that does not allow the jets to remain attached to the wall at this injection condition. This is even worst at the highest tested $M_{1}$ of 2.0 when a complete jet liftoff takes place. As a consequence, practically no traces of coolant can be detected at the wall for the largest tested injection condition.

Focusing on the downstream PS holes, according to previous tests, a blowing rate increase always translates into a thermal protection improvement, that in the limited range of $M_{1}$ values tested in this research is quite small. Previous experiments involved a wider range of injection conditions, up to $M_{1}=4.0$. As typical values of blowing ratio for this cooling scheme are lower, injection conditions were limited here to about 2.0.

Trying to improve the cooled area, two more holes were manufactured on the platform and tested under similar injection conditions (Fig. 9 - 7 hole cooling scheme at $M_{1}$ about 1.1, 1.5 and 2.1 for both PS holes and new holes). The cooling scheme with two more holes show a non negligible increase of the thermal protection for the low injection condition. More coolant now reaches the suction side of the adjacent blade, even if again it is not allowed to cross the 3D separation lines. Its momentum is not enough to counteract the passage vortex washing activity, resulting in a quick decay. But as soon as $M_{1}$ is increased up to 1.5 or even up to 2.1, jet lift off takes place again, resulting in a lack of thermal protection. In particular, the last hole is characterized by a so high coolant momentum that no trace of this jet on the wall can be seen, both for $M_{1}=1.5$ and 2.1.

This is due again to the high injection angle and to the hole location very close to the passage vortex three dimensional separation lines. The latter does not allow the coolant to travel across the passage, resulting in an abrupt decrease of effectiveness.

Finally, it has to be noted that test duration was higher for the 5-hole cooling scheme, and particularly for the low injection condition, resulting in a higher influence of heat conduction close to hole location. This explain the differences between the effectiveness distribution downstream the PS holes.

In order to better enlighten coolant jets behavior downstream of the new injection holes, film cooling effectiveness data have been extracted along the interplatform line (see Fig. 3). Figure 10 compares such data belonging to the two tested hole numbers at variable $M_{1}$. Data are plotted against the $S$ coordinate, i.e. a coordinate tangent to the inter-platform line, whose origin is at the filleted leading edge plane and that it is normalized using its value at the filleted trailing edge plane. The first hole is located approximately at $S / S_{T E}=0.14$, while the fifth is at about 0.32 and the seventh at 0.41 .

First considering the 5-hole configuration (Fig. 10a), according with the previous analysis, best performance is attained at the lowest tested injection rate of about 1.2. $\eta / \eta_{\max }$ values as large as 0.38 are reached close to the 5th hole, also due to a cumulative effect. Increasing the blowing condition up to $1.6 \quad \eta / \eta_{\max }$ decreases down to about 0.2 as a maximum, also limiting its thermal protection along the inter-platform separation line. Increasing $M_{1}$ up to the maximum tested value of 2.0 results in a further reduction of 


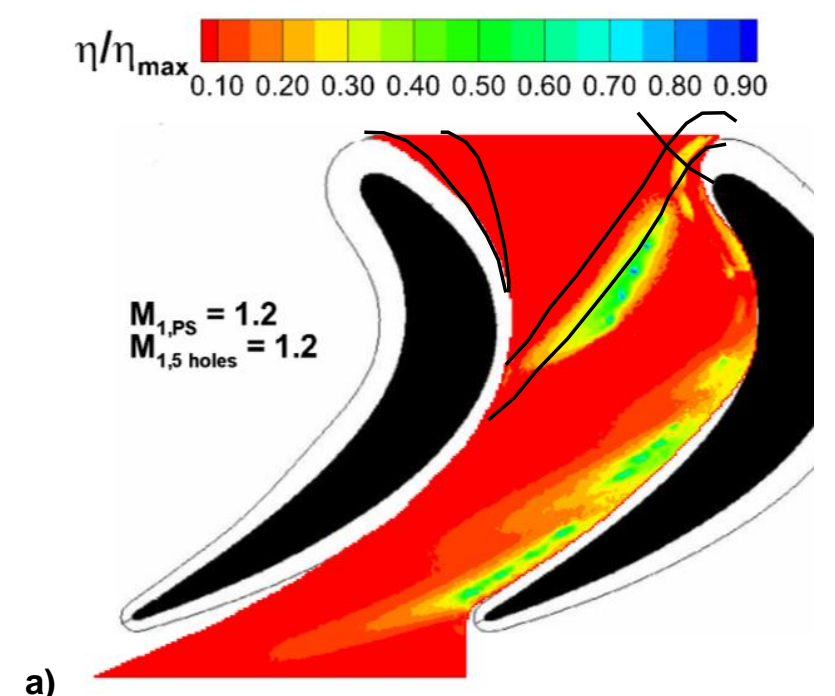

a)

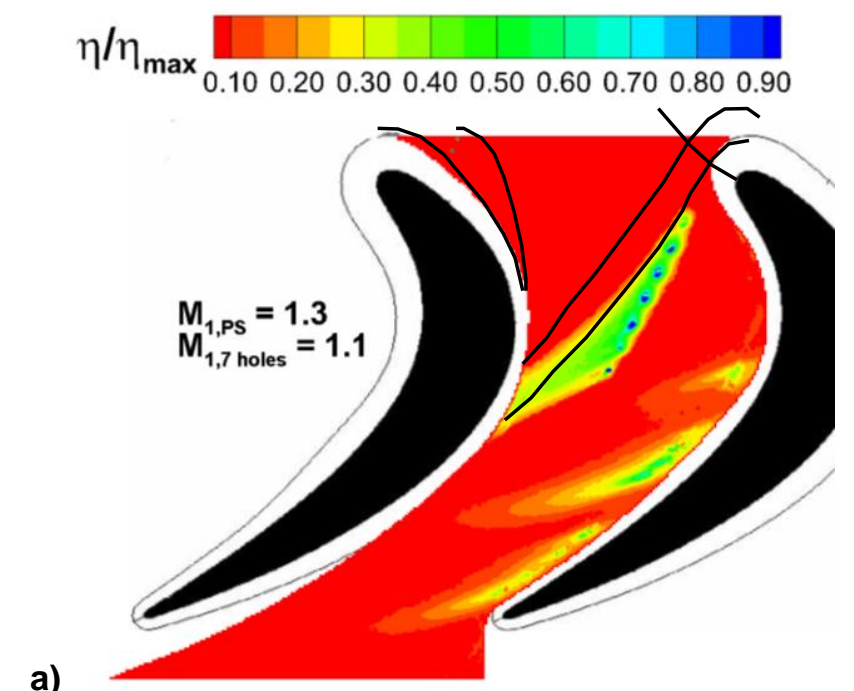

b)
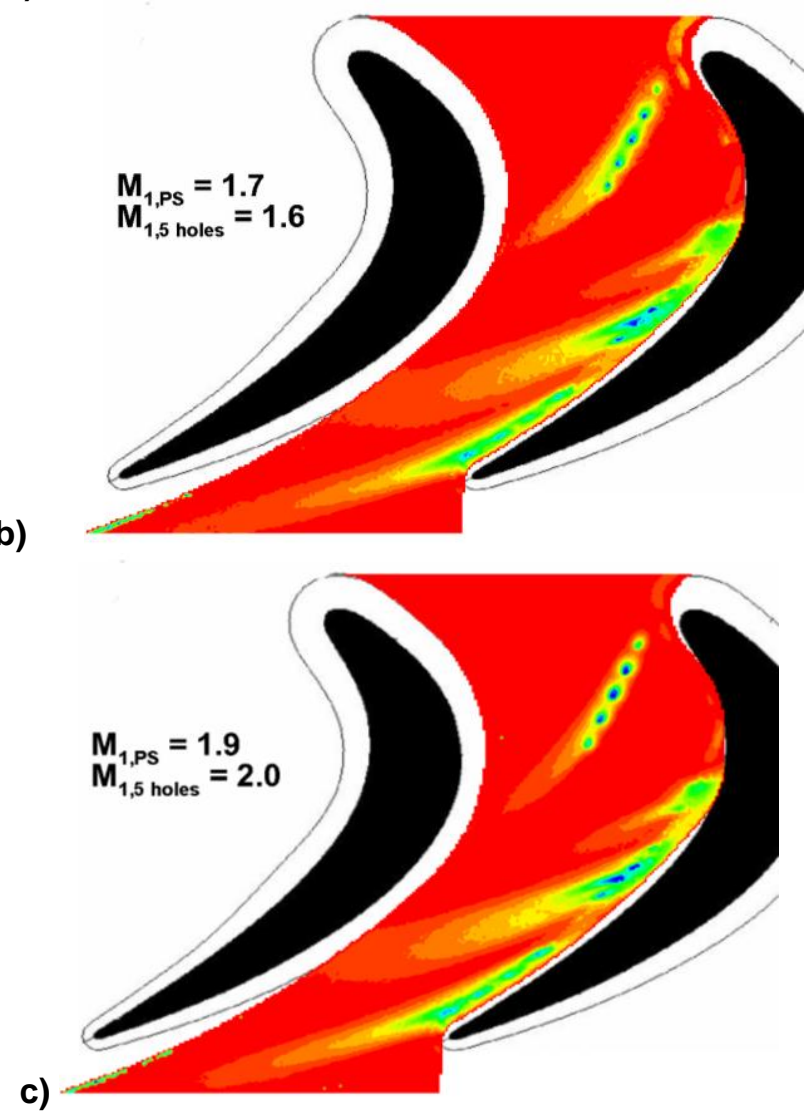

Figure 8. Film cooling effectiveness distributions for the 5 hole scheme and variable injection conditions.

$\eta / \eta_{\max }$ to about 0.1 , but also in a shift of the cooled region. The high momentum coolant seems to be less influenced by the end wall cross flow, resulting in a coolant trace more aligned to the platform separation line. Moreover, the jet coming from the last hole is completely detached from the wall, so cooling here is only due to the preceding holes.

a)

b)

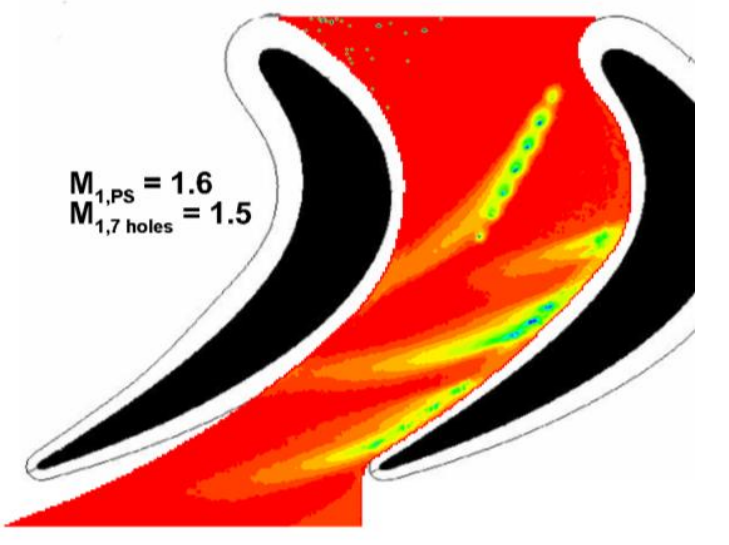

c)

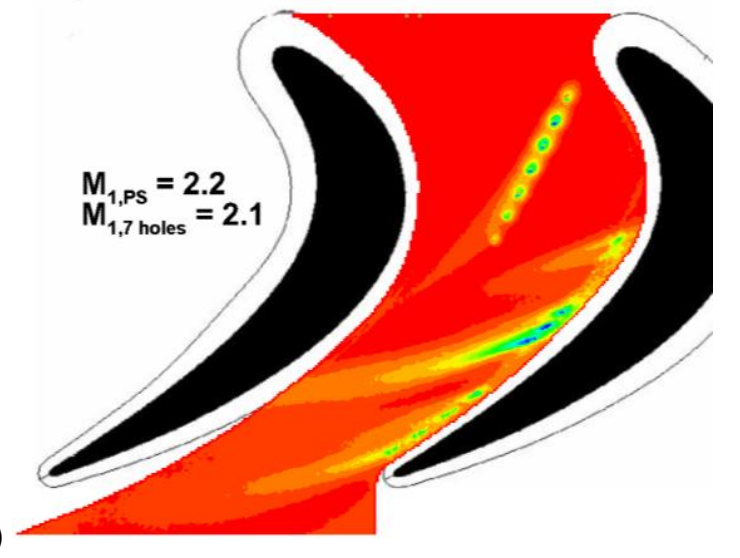

Figure 9. Film cooling effectiveness distributions for the 7 hole scheme and variable injection conditions.

Adding two holes along the same platform separation line slightly increases the peak effectiveness at the lowest tested condition $\left(M_{1}=1.1\right)$ about 0.4. In fact, the same, or about the same $M_{1}$ value allows the 7-hole configuration to discharge a higher mass flow when compared to the 5-hole scheme. This higher mass flow is discharged through the last two holes with a high momentum: this is 
responsible for the high effectiveness levels observed where the last hole is located (the 7th hole is located at about $S / S_{T E}=0.41$ ). Increasing the injection condition results in a progressive reduction of thermal protection that, for the largest tested $M_{1}$ of 2.1 , is even worse than the 5-hole scheme (below 0.1). In fact the 7th hole does not contribute to cool the platform. Whatever the injection condition, the 7holes configuration allows to cool a slightly wider platform region, when compared with the 5-hole scheme.

a)
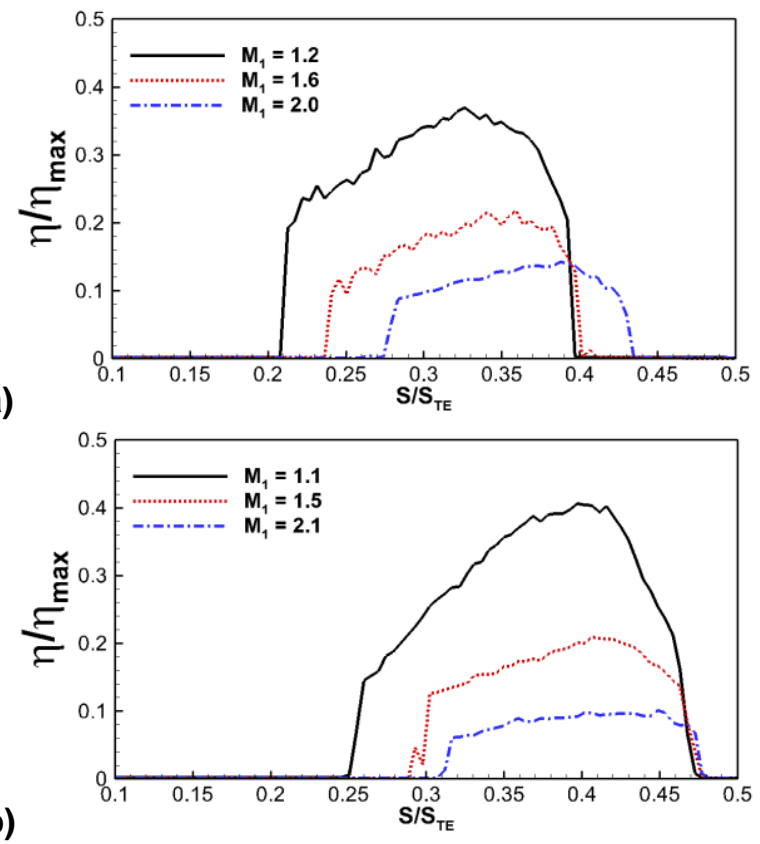

Figure 10. $\eta$ distributions along the inter-platform separation line: a) 5 holes and b) 7 holes configuration.

As a final comment, both the investigated schemes get the best performance at a relatively low injection rate of about $M_{1}=1.0$. The cooling scheme with 5 holes shows a lower cooled area, if compared to the 7 hole scheme. Larger blowing rates always result in a loss of performance: the high injection angle quickly promotes jet lift off. This is exactly the opposite of what happens rear on the channel due to the PS cooling holes. Here, the low injection angle $\left(11.5^{\circ}\right)$ allows the high momentum fluid to remain attached to the wall increasing the blowing rate.

\section{CONCLUSIONS}

An experimental investigation on the thermal effects related to the introduction of new holes inside of the passage to cool the inter-platform gap region has been carried out. From the presented results the following conclusions can be drawn:
- Due to the very small injected mass flow, no significant modifications of secondary flows configuration and aerodynamic losses take place.

- Whatever the hole number, best thermal protection is attained at low coolant blowing ratios $M_{1}$ of about 1.0.

- A blowing ratio increase always result in a worsening of thermal protection due to jet lift off from the wall caused by the high injection angle.

- The 7-hole configuration allows the cooling of a larger platform region, extending up to the suction side of the following blade, up to about $0.5 c_{a x}$.

\section{ACKNOWLEDGMENTS}

The authors wish to thank Ing. P. Epis and L. Padovan for their appreciated support.

\section{REFERENCES}

[1] M.F. Blair. An Experimental Study of Heat Transfer and Film Cooling on Large-Scale Turbine Endwalls. J. Heat Transfer, 96:524-529, 1974.

[2] R.P. Roy, K.D. Squires, M. Gerendas, S. Song, W.J. Howe and A. Ansari. Flow and Heat Transfer at the Hub Endwall of Inlet Vane Passages - Experiments and Simulations. ASME Paper 2000-GT-198, 2000.

[3] R.A. Oke and T.W. Simon. Film Cooling Experiments with Flow Introduced upstream of a First Stage Nozzle Guide Vane through Slots of Various Geometries. ASME Paper 2002-GT-30169, 2002.

[4] R.A. Oke, T.W. Simon, S.W. Burd and R. Vahlberg. Measurements in a Turbine Cascade over a Contoured Endwall: Discrete Hole Injection of Bleed Flow. ASME Paper 2000-GT-214, 2000.

[5] M.J. Jabbari, K.C. Marston, E.R.G. Eckert and R.J. Goldstein. Film Cooling of the Gas Turbine Endwall by Discrete-Hole Injection. J. of Turbomach, 118:278284, 1996.

[6] S. Friedrichs, H.P. Hodson and W.N. Dawes. Distribution of Film-Cooling Effectiveness on a Turbine Endwall Measured with the Ammonia and Diazo Technique. J. Turbomach, 118: 613-621, 1996.

[7] F. Kost and M. Nicklas. Film-Cooled Turbine Endwall in a Transonic Flow Field: Part I - Aero-dynamic Measurements. ASME Paper 2001-GT-0145, 2001.

[8] M. Nicklas. Film-Cooled Turbine Endwall in a Transonic Flow Field: Part II - Heat Transfer and Film-Cooling Effectiveness. ASME Paper 2001-GT0146, 2001.

[9] D.G. Knost and K.A. Thole. Adiabatic Effectiveness Measurements of Endwall Film-Cooling for a First Stage Vane. ASME Paper 2004-GT-53326, 2004.

[10] G. Barigozzi, G. Benzoni, G. Franchini and A. Perdichizzi. Fan-shaped Hole Effects on the AeroThermal Performance of a Film Cooled Endwall. J. Turbomach. 128:43-52, 2006. 
[11] M.F. Blair. An Experimental Study of Heat Transfer in a Large-Scale Turbine Rotor Passage. J. Turbomach, 116:1-13, 1994.

[12] H.-P. Wang, S.J. Olson, R.J. Goldstein and E.R.G. Eckert. Flow Visualization in a Linear Turbine Cascade of High Performance Turbine Blades. J. Turbomach, 119:1-8, 1997.

[13] R.J. Goldstein and R.A. Spores. Turbulent Transport on the Endwall in the Region Between Adjacent Turbine Blades. J. Heat Transfer, 110: 862-869, 1988.

[14] Z. Gao, D. Narzary and J.C. Han. Turbine Blade Platform Film Cooling with Typical Stator-Rotor Purge Flow and Discrete-Hole Film Cooling. ASME Paper 2008-GT-50286, 2008.

[15] D. Narzary. Experimental Study of Gas Turbine Blade Film Cooling and Heat Transfer. PhD Dissertation, Texas A\&M University, August 2009.

[16] M. Papa, V. Srinivasan, and R.J. Goldstein. Film Cooling Effect of Rotor-Stator Purge Flow on Endwall Heat/Mass Transfer. J. Turbomach, 134: 041014:1-8, 2012.

[17] G. Barigozzi, G. Franchini, A. Perdichizzi, M. Maritano and R. Abram. Influence of Purge Flow Injection angle on the aero-thermal performance of a rotor blade cascade. J. Turbomach, 136:0410121:10, 2014.

[18] M. Pau, G. Paniagua, D. Delhaye, A. de la Loma and P. Ginibre. Aerothermal Impact of Stator-Rim Purge Flow and Rotor-Platform Film Cooling on a Transonic Turbine Stage. J. Turbomach. 132:021006, 2010.

[19] A.Suryanarayanan, S. Mhetras, M. T. Schobeiri and J.C. Han. Film Cooling Effectiveness on a Rotating Blade Platform. J. Turbomach, 131: 011014:1-12, 2009.

[20] A. Suryanarayanan, B. Ozturk, M.T. Schobeiri and J.C. Han. Film-Cooling Effectiveness on a Rotating Turbine Platform Using Pressure Sensitive Paint Technique. J. of Turbomach, 132:041001, 2010.

[21] L.M. Wright, S.A. Blake and J.C. Han. Film Cooling Effectiveness Distributions on a Turbine Blade Cascade Platform With Stator-Rotor Purge and Discrete Film Hole Flows. J. Turbomach,130: 031015, 2008.

[22] H. Yang, Z. Gao, H.C. Chen, J.C. Han and M.T. Schobeiri. Prediction of Film Cooling and Heat Transfer on a Rotating Blade Platform With StatorRotor Purge and Discrete Film-Hole Flows in a 1 1/2 Turbine Stage. J. Turbomach, 131:041003, 2009.

[23] G. Barigozzi, F. Fontaneto, G. Franchini, A. Perdichizzi, M. Maritano and R. Abram. Influence of Coolant Flow Rate on Aero-Thermal Performance of a Rotor Blade Cascade with Endwall Film Cooling. J. Turbomach, 134:051038-1:8, 2012.

[24] G. Barigozzi, S. Ravelli, M. Maritano and R. Abram. Computational predictions of aero-thermal performance of a turbine blade cascade with endwall film cooling. ASME Paper GT2012-69049, 2012.

[25] C. Camci, K. Kim., S.A. Hippensteele. A New Hue Capturing Technique for the Quantitative Interpretation of Liquid Crystal Images Used in Convective Heat Transfer Studies. J. of Turbomach. 114:765-775, 1992. 